\title{
Optimal Sizing of Electric Multirotor Configurations
}

\author{
Giulio Avanzini ${ }^{1, *}$, Emanuele L. de Angelis ${ }^{2}$, Fabrizio Giulietti ${ }^{2}$, and Edmondo Minisci ${ }^{1}$ \\ ${ }^{1}$ University of Salento, Dep. of Engineering for Innovation, 73100 Lecce, Italy \\ ${ }^{2}$ University of Bologna (Forlì Campus), Dep. of Industrial Engineering, 47121 Forlì, Italy \\ ${ }^{3}$ University of Strathclyde, Dep. of Mechanical and Aerospace Engineering, G1 1XJ Glasgow, UK
}

\begin{abstract}
A sizing tool for the definition of the configuration of electrically powered multirotor platforms is developed, which accounts for a realistic battery discharge model. The tool is developed to provide the community with the possibility of deriving the best configuration for performing a given task, while accounting for specific constraints and performance requirements. An evolutionary algorithm is used for searching the design space and to identify feasible designs with optimal performance in terms of maximum hovering time on the target and payload weight fraction.
\end{abstract}

\section{Introduction}

Electric multirotor vehicles are becoming increasingly popular in many civil applications as well as leisure activities [1]. Regulatory authorities allow only for Visual-Line-of-Sight (VLOS) operations. Nonetheless, the possibility of Beyond-Visual-Line-of-Sight (BVLOS) operations has been studied and demonstrated in tests performed by industry and academia, thanks to the combined use of advanced control systems and filtering techniques [2,3], where virtual and augmented reality can be adopted to increase the level of situational awareness of a remote pilot [4]. Demanding autonomous tasks, such as the use of a fleet of autonomous vehicles, with collision avoidance capabilities, have also been studied [5].

In spite of their increasing popularity and an improved knowledge of their dynamics [6], manufacturing of this class of vehicles still relies more on experience than on a systematic design approach, starting from a set of consistent mission requirements and based on a possibly simple, yet theoretically sound, modelling approach. The procedure for preliminary sizing of fixed wing aircraft is well established [7-9]. The procedure is based on a combination of three elements:

1. definition of the configuration as a function of aircraft class and expected mission;

2. statistical regression of relevant sizing data for existing aircraft in the same class;

3. a limited number of data on aerodynamics and propulsion which allows for a reasonable performance estimate for the sizing mission.

Performance estimate requires a set of equations, such as Breguet equations for endurance and range, which relates aircraft data with expected operational capabilities (total distance covered for a given amount of fuel, climb speed, efficiency during cruise, etc.). Multirotor platforms come in a variety of configurations, the planar quadrotor being the most common

\footnotetext{
*Corresponding author: giulio.avanzini@unisalento.it
} 
one, but a relation between configuration and mission in not yet established. At the same time, statistical data for existing electrically powered fixed- and rotary-wing aircraft show a very poor correlation. Finally, the estimate of expected performance based on a (possibly limited) set of aircraft parameters has been developed only recently for fixed-wing electrical aircraft $[10,11]$. The latter works allowed for a preliminary sizing of the battery pack for an electrically powered fixed wing aircraft [1], but with the exception of some studies on hybrid helicopter configurations [12], there is little work available on sizing electric single main rotor helicopter and multirotor vehicles. In a recent study [13], a parametric analyses for identifying best endurance configurations of electric rotary wing aircraft was performed, which resulted in some surprising results (e.g. the weight of the battery pack should be $2 / 3$ of the total weight of the vehicle for maximizing flight time). This study was performed adopting a battery discharge model based on Peukert's law [14], which assumes a constant current and voltage during the discharge process. Moreover, only the maximum endurance in hovering condition was considered for the battery pack sizing procedure, in the absence of one or more cruise legs to a distance target area.

The present paper aims at implementing a more sophisticated sizing approach, where (1) a recent realistic battery discharge model presented in [1] is adopted, which accounts for voltage decrease during a constant power discharge process (resulting in a steadily increasing current drawn), and (2) a more realistic mission profile is adopted, which accounts for the possibility of monitoring a far site. The model will be coupled with a multi-objective memetic evolutionary algorithm for mixed integer problems [15], to identify the best configuration(s), while providing systematic information on the best compromise between competing objectives (e.g. payload fraction vs loiter time on the operational area), subject to constraints on performance, geometry, and structure/masses.

The mission scenario is the inspection of a remote site, where the vehicle is required to fly to the target area, spend as much time as possible loitering on the site, and fly back to the initial point. Distance flown and payload weight are problem parameters. When distance is zero, a loitering mission in VLOS condition is considered. Prescribed payload weight for different (possibly zero) distance to target area and prescribed distance for various payloads will be considered. The sizing tool assists the designer in assessing the best configuration, based on actual operator needs, while sizing the battery pack for maximizing mission duration. Constraints on geometrical size and weight can be introduced in order to meet regulatory requirements and/or other specifications.

In the next paragraph, a multirotor model is introduced, for the determination of power required for flying the vehicle at different airspeed. An empirical constant power battery discharge model is outlined, which can accommodate for an estimate of discharge time for a step variation of power drawn from the battery pack. The optimization problem is stated in Section 3, where some details on the optimization algorithm adopted are also provided, The results obtained are discussed in Section 4. A section of conclusions ends the paper.

\section{Multirotor model}

\subsection{Geometry and weights}

Figures 1.a and b depict the schemes of two classes of multirotor platform, namely, a quadrotor configuration, in a planar configuration (Fig. 1.a), and a hexacopter with three pairs of counter-rotating rotors (Fig. 2.b). The number of rotors for most multirotor platforms is an even number between 4 and 8 . A higher number of rotors is employed only for peculiar configurations with specific needs. Also, a combination of single and coaxial rotors can be used. Both these options will not be considered in this study, where only conventional quadcopters, exacopters and octocopters will be considered. 


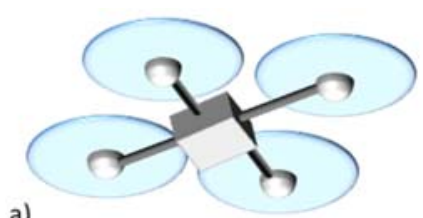

a)

Fig. 1. Multirotor planar (a) and coaxial (b) configurations.
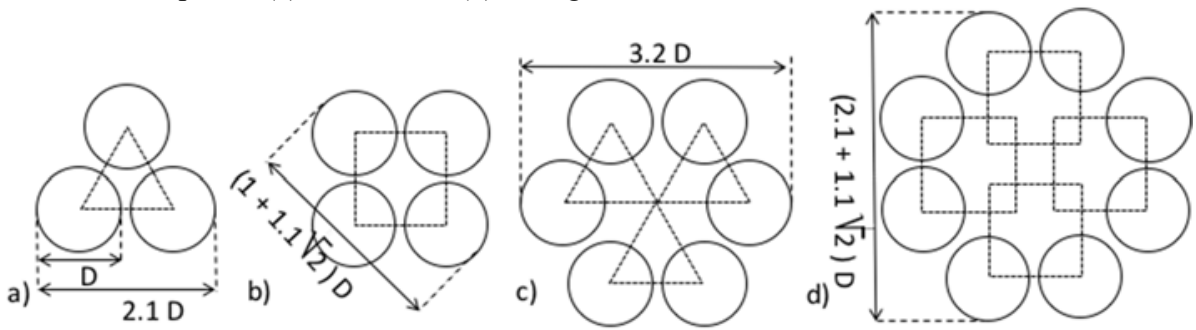

Fig. 2. Configurations: coaxial exacopter (a); planar quadcopter or coaxial octocopter (b); planar exacopter (c) and octocopter (d).

When the number of rotors is equal to six or higher, a coaxial configuration becomes available, which allows for a more compact size, for the same takeoff weight, at the expenses of a minor efficiency of the propulsion system, where the power consumption of counterotating rotors becomes higher than that of a single rotor producing the same thrust. A total of five different multirotor configurations are thus considered in this study, namely 3 planar ones, with 4,6 or 8 rotors, and 2 coaxial ones, with 6 or 8 rotors. A shape coefficient defines the maximum width of the vehicle, $l$, as a function of propeller diameter $d$, number of rotors, $N_{\text {rot }}$, and configuration, as indicated in Fig. 2. The shape coefficient $\sigma$, such that $l=\sigma d$, is derived assuming that rotor separation is equal to $10 \%$ of rotor diameter $d$.

Vehicle weight is obtained as the sum of 5 terms,

$$
W_{T O}=W_{p l}+W_{s t r}+W_{s y s}+W_{\text {prop }}+W_{b a t}
$$

namely payload weight, $W_{p l}$, weight of the structure (central structure, landing gear, rotor supports, etc.), $W_{s t r}$, weight of electronic boards and systems, $W_{s y s}$, weight of the propulsion system, $\$ W_{\text {prop }}$, and weight of the battery pack, $W_{\text {bat }}$.

The weight of the propulsion system is obtained as the sum of the weights of electrical engine, regulator and propeller assembly times the number of rotors, $N_{\text {rot }}$, that is

$$
W_{\text {prop }}=N_{\text {rot }}\left(W_{\text {eng }}+W_{\text {reg }}+W_{\text {rot }}\right)
$$

Structure weight is given by

$$
W_{s t r}=W_{s t r, 0}+\left(1-K_{C R} w\right) N_{\text {rot }} W_{\text {sup }}
$$

where $W_{s t r, 0}$ is the weight of the central body (including the undercarriage), whereas $W_{\text {sup }}$ is the weight of the rotor support and mount. The coefficient $K_{C R}$ accounts for the possibility of counter-rotating rotors, being $K_{C R}=0$ for planar configurations and $K_{C R}=1$ for counterrotating ones. In this latter case, the number of supports is halved, with respect to the number of rotors. Ideally, a percentage $w=0.5$ of support weight is saved, but each support must withstand a larger load (in general twice the load of a single rotor for a planar configuration), which means that the fraction of support weight saved is, in general, $0<w<0.5$.

Finally, payload weight $W_{p l}$ and weight of electronic boards and systems $W_{s y s}$ are assumed known, whereas the weight of the battery pack $W_{b a t}$ is one of the design parameters. The total weight $W_{T O}$ may be required to not exceed a possibly prescribed threshold. 


\subsection{Aerodynamic model and required power}

Forces acting on a multirotor vehicle are vehicle weight, $W_{T O}$, total rotor thrust, $T$, and aerodynamic drag, $D$. Drag acts in the direction opposite to vehicle velocity relative to the air, $V$. It is developed mainly by body, strut and undercarriage. The value of the equivalent flat plate area is $A_{e f p}=S C_{D}$, where $C_{D}$ is the drag coefficient and $S$ a reference area. For the sake of simplicity, $A_{e f p}$ is assumed independent of vehicle angle of attack, which is typically negative in forward flight. This assumption is consistent for a preliminary sizing process, when the actual shape of the vehicle is not yet still known, and a precise estimate of aerodynamic coefficients not available. Drag is thus estimated as $D=0.5 \rho V^{2} A_{e f p}$.

The equilibrium in hovering requires that $T=W_{T O}$, whereas in forward flight it is

$$
-T \sin \alpha-D=0 ; T \cos \alpha-W_{T O}=0
$$

In this latter case $T^{2}=W_{T O}{ }^{2}+D^{2}$ and $\tan \alpha=-D / W$. Each rotor produces a thrust assumed proportional to the square of its rotational rate, $\Omega_{i}, i=1,2, \ldots, N_{\text {rot }}$, that is, $T_{i}=k_{\Omega} \Omega_{i}{ }^{2}$. At equilibrium $T_{i}=T / N_{\text {rot }}$, hence the value of $\Omega_{i}$ is derived from the considered trim state.

The power required by the vehicle can be decomposed into three contributions,

$$
P_{R}=P_{a v}+P_{p l}+P_{\text {prop }}
$$

where power required by avionics and payload $\left(P_{a v}\right.$ and $\left.P_{p l}\right)$ are assumed known, whereas the power required by the propulsion system, $P_{\text {prop }}=N_{\text {rot }} P_{r o t}$, with $P_{r o t}=k_{P} \Omega_{i}{ }^{3}$, depends on number of rotors and trim state, trough $\Omega_{i}$. In forward flight rotor power increases as a function of the advance ratio, $\mu=V /(\Omega R)$, where $R=d / 2$ is rotor radius. Letting $P_{\text {rot }, 0}$ be the power required in hovering at $\mathrm{V}=0$ for delivering the required amount of thrust, one has that

$$
P_{r o t}=P_{r o t, 0}\left(1+3 \mu^{2}\right)\left(1+0.22 K_{C R}\right)
$$

where the $0.22 K_{C R}$ increment accounts for a rotor power increment in coaxial configurations.

\subsection{Battery discharge model}

In an ideal discharge process, the voltage of the battery is assumed constant and the charge extracted from the battery per unit time proportional to the current $i$. As a matter of fact, the intensity of the current drawn from a battery affects its actual capacity. In general, the charge drawn from the battery is reduced for currents higher than the nominal one because of higher internal losses. This phenomenon is known as Peukert's effect. For a constant current discharge process, Peukert proposed a simple law [14], $t_{D} i^{k}=$ const, where Peukert's exponent $k<1$ characterizes the class of batteries. Higher values of $k$ indicate a lower battery efficiency at higher currents.

Peukert's law provides a better approximation for battery discharge time than the ideal model. Nonetheless, the hypothesis of a constant current discharge process is often far from the actual operational use of a battery pack, when one considers that voltage also decreases during the discharge, thus affecting the relation between current drawn for a given power. In many applications, a constant power discharge process is often a more accurate hypothesis. Reference [1] describes in some detail an empirical battery discharge model experimentally obtained for constant power discharge processes by means of a controllable electric load. The discharge time is written in the form

$$
t_{D}=\delta \mathrm{P}_{D}^{\varepsilon} C_{0}^{\beta}
$$


where $P_{D}$ and $C_{0}$ are power discharged from the battery pack and its nominal capacity at full charge, respectively. The coefficients $\delta, \varepsilon$, and $\beta$ are empirically derived for the considered class of batteries cells. An ideal discharge process is obtained for $\delta=v, \varepsilon=-1$ and $\beta=1$.

The model described in Eq. (1) refers to a constant power discharge process. For a mission that requires segments such that power undergoes a limited number of step variations it is possible to extend the model to time-varying values of $P_{D}$. Assume that the discharge process at power $P_{D 1}$ is performed for a time $t_{1}<t_{D}$, for the same power level. It is possible to assume that a residual nominal capacity $C_{1}$ is left, such that $t_{D}-t_{1}=\delta \mathrm{P}_{D 1}{ }^{\varepsilon} C_{1}{ }^{\beta}$, that is,

$$
C_{1}=\left[\left(t_{D}-t_{1}\right) /\left(\delta \mathrm{P}_{D 1}^{\varepsilon}\right)\right]^{1 / \beta}
$$

The residual discharge time at a different power level $P_{D 2}$ thus becomes $t_{D, \text { res }}=\delta \mathrm{P}_{D 2}{ }^{\varepsilon} C_{0}{ }^{\beta}$. If the discharge time at power $P_{D 2}$ is $t_{2}<t_{D, \text { res }}$, the process can be iterated, and a new residual nominal capacity is evaluated in the form

$$
C_{2}=\left[\left(t_{D, r e s}-t_{2}\right) /\left(\delta \mathrm{P}_{D 2}{ }^{\varepsilon}\right)\right]^{1 / \beta}
$$

\section{Optimization of multirotor configurations: problem statement}

\subsection{Configurations}

As outlined above, 5 different (and most common) multirotor platform configurations are considered in this study, which are numbered as indicated in Tab. 1. Also, a set of 15 different engine/regulator/propeller assemblies of various diameters and nominal power, listed in Table 1, are considered for the sizing process. Two indexes are introduced among design variables, one which varies between 1 and 5, for the configuration, and the second between 1 and 15, for selecting the propulsion system. Vehicle empty weight (e.g. the weight of the multirotor without the battery pack) is determined from these two indexes, assuming payload weight is known, $W_{E}=W_{p l}+W_{s t r}+W_{s y s}+W_{p r o p}$. The weight of the battery pack is then sized as a fraction $f$ (possibly greater than one) of the empty weight, so that the takeoff weight is expressed as

$$
W_{T O}=W_{E}+W_{b a t}=(1+f) W_{E}
$$

\subsection{Sizing missions and optimization variables}

In a local survey mission scenario, the vehicle is operated in Visual Line Of Sight (VLOS), under constant supervision by its remote pilot. For a relatively small altitude gain and very low airspeed, the flight condition resembles a hovering state, in which total thrust delivered by rotors almost exactly balances vehicle weight. A constant power discharge process thus represents reasonably well the use of battery energy. Assuming that the whole energy stored in the battery is used prior to landing the vehicle, merit functions are hovering time, $t_{\mathrm{H}}$, and payload weight fraction $W_{p l} / W_{T O}$. The design variables are two integers, namely the configurations indices, and a positive real number, that is, the battery weight fraction, $f$.

A second scenario is tackled, where the multirotor platform is expected to operate Beyond Visual Line Of Sight (BVLOS operations), in which case a transfer distance to a target area of operations $d_{\mathrm{TRG}}$ needs to be covered from the launch site. The vehicle is expected to land on the departure site, thus completing a return trajectory. Provided that the discharge model adopted is nonlinear, the best airspeed may be in general different for the transfer to and from the operation site. A total of 3 real design variables are thus present, namely the speed for the two cruise segments, $V_{1}$ and $V_{2}$, and the battery weight fraction, $f$. As in the previous case, two integer design variables are also present, for selecting configuration and propulsion system. The distance $d_{T R G}$ between departure site and target area of operations is assumed known. A parametric analysis for different values of $d_{T R G}$ is performed. The merit functions 
are hovering time $t_{H}$ at the target site and the payload weight fraction, $W_{p l} / W_{T O}$. The former is evaluated by assuming that two constant power transfer legs are flown with a duration equal to $t_{1}=d_{T R G} / V_{1}$ and $t_{2}=d_{T R G} / V_{2}$. Assuming that $P_{1}$ and $P_{2}$ represent required power during the transfer legs flown at airspeed equal to $V_{1}$ and $V_{2}$, respectively, one has that $C_{1}{ }^{\beta}=$ $\left(t_{D 1}-t_{1}\right) /\left(\delta \mathrm{P}_{D 1}{ }^{\varepsilon}\right)$, where $t_{D 1}$ is the total discharge time available at power $P_{1}$, and $C_{2}{ }^{\beta}=t_{2} /$ $\left(\delta \mathrm{P}_{D 1}{ }^{\varepsilon}\right)$. The resulting hovering time at the target operation site is thus equal to

$$
t_{H}=\delta \mathrm{P}_{H}^{\varepsilon}\left(C_{1}^{\beta}-C_{2}^{\beta}\right)
$$

where $P_{H}$ is the power required at hovering.

Table 1. List of multirotor platform configurations.

\begin{tabular}{|c|c|c|c|}
\hline $\begin{array}{c}\text { Configuration } \\
\text { index }\end{array}$ & $\begin{array}{c}\text { No. of } \\
\text { rotors } \boldsymbol{N}_{\text {rot }}\end{array}$ & $\begin{array}{c}\text { Planar (P) vs } \\
\text { coaxial (C) }\end{array}$ & $\begin{array}{c}\text { Shape } \\
\text { factor } \boldsymbol{\sigma}\end{array}$ \\
\hline 1 & 4 & $\mathrm{P}$ & 2.56 \\
\hline 2 & 6 & $\mathrm{P}$ & 3.20 \\
\hline 3 & 6 & $\mathrm{C}$ & 2.10 \\
\hline 4 & 8 & $\mathrm{P}$ & 3.66 \\
\hline 5 & 8 & $\mathrm{C}$ & 2.56 \\
\hline
\end{tabular}

Table 2. Electric motor characteristics.

\begin{tabular}{|c|r|r|r|r|r|r|r|r|}
\hline $\begin{array}{c}\text { Eng. - rot. } \\
\text { index }\end{array}$ & $\begin{array}{c}\text { Diameter } \\
d[\mathrm{in}]\end{array}$ & $\begin{array}{c}\text { Pitch } \\
\theta[\mathrm{deg}]\end{array}$ & $\begin{array}{c}\text { Current } \\
i[\mathrm{~A}]\end{array}$ & $\begin{array}{c}\text { Power } \\
P_{\text {eng }}[\mathrm{W}]\end{array}$ & $\begin{array}{c}\text { Thrust } \\
T_{\text {eng }}[\mathrm{kg}]\end{array}$ & $\begin{array}{c}\text { RPM } \\
\Omega\end{array}$ & Efficiency & $\begin{array}{c}\text { Mass } \\
{[\mathrm{kg}]}\end{array}$ \\
\hline 1 & 26 & 8.5 & 1.6 & 35.52 & 0.710 & 1200 & 19.95 & 0.242 \\
\hline 2 & 27 & 8.8 & 1.7 & 37.34 & 0.770 & 1160 & 20.40 & 0.242 \\
\hline 3 & 28 & 9.2 & 1.8 & 39.96 & 0.860 & 1130 & 21.52 & 0.242 \\
\hline 4 & 29 & 9.5 & 2.0 & 44.4 & 0.930 & 1100 & 20.95 & 0.242 \\
\hline 5 & 26 & 8.5 & 2.3 & 51.06 & 0.980 & 1400 & 19.19 & 0.242 \\
\hline 6 & 27 & 8.8 & 2.6 & 57.72 & 1.040 & 1350 & 18.02 & 0.242 \\
\hline 7 & 28 & 9.2 & 3.0 & 66.6 & 1.100 & 1300 & 16.52 & 0.242 \\
\hline 8 & 29 & 9.5 & 3.2 & 71.04 & 1.330 & 1300 & 18.72 & 0.242 \\
\hline 9 & 26 & 8.5 & 4.4 & 97.68 & 1.420 & 1700 & 14.54 & 0.242 \\
\hline 10 & 27 & 8.8 & 4.9 & 108.78 & 1.590 & 1650 & 14.62 & 0.242 \\
\hline 11 & 28 & 9.2 & 5.5 & 122.1 & 1.820 & 1600 & 14.91 & 0.242 \\
\hline 12 & 29 & 9.5 & 6.0 & 133.2 & 1.930 & 1560 & 14.49 & 0.242 \\
\hline 13 & 30 & 10.5 & 6.2 & 148.8 & 2.132 & 1560 & 14.33 & 0.273 \\
\hline 14 & 28 & 9.2 & 8.1 & 194.4 & 2.401 & 1953 & 12.35 & 0.273 \\
\hline 15 & 29 & 9.5 & 9.1 & 218.4 & 2.627 & 1899 & 12.03 & 0.273 \\
\hline
\end{tabular}

\subsection{Sizing missions and optimization variables}

A solution is acceptable only if $t_{H}>0$. Other operational constraints may be present, such as a limit on takeoff weight, $W_{T O}<W_{\max }$, because of regulatory issues, in order to attain a weight which allows for developing a vehicle in a class with simpler certification requirements. Another constraint may be induced by requirements on vehicle size $l=\sigma d<l_{\max }$, where $l$ may be required to remain below a given limit for accessing confined spaces and/or ease of storage during transport. A constraint is also introduced on rotor rate, $\Omega$, assuming that manufacturer rotor performance in terms of thrust and power for a reference rotor rate $\Omega_{0}$ are 
provided at $75 \%$ throttle level. Rotor rate is thus bounded, such that $\Omega<1.1 \Omega_{0}$. These constraints are introduced in the sizing tool, but different ones may be easily added, if needed.

\subsection{Numerical technique}

The search method used in this work is a memetic evolutionary algorithm, the MultiPopulation Inflationary Differential Evolution Algorithm (MP-IDEA) [15], here adapted to deal with mixed-integer and constrained problems. Constraints are dealt with by means of a penalty approach that separates objectives and constraints. The objective function (to be minimized) is formulated as

$$
F(\mathbf{x})=\left\{\begin{array}{l}
f(\mathbf{x}), \text { is feasible } \\
\Delta_{f}+\sum_{i=1}^{m} \max \left(0, g_{i}(\mathbf{x})\right)+\delta_{f} \operatorname{sign}\left(\sum_{i=1}^{m} \max \left(0, g_{i}(\mathbf{x})\right)\right), \text { otherwise }
\end{array}\right.
$$

where $\mathbf{x}$ is the $N_{d}$-dimensional design vector, $f(\mathbf{x})$ is the objective function, $g_{i}(\mathbf{x})<0$ is the $i$-th constraint function, $i=1,2, \ldots, m, \Delta_{f}$, is a constant higher than the worst value of $f$, and $\delta_{f}>1$ is a constant. The algorithm works with continuous and integer variables normalized in the hypercube $[0,1]^{\mathrm{Nd}}$. When re-mapped to real values, integer parameters are rounded to the closest integer. Results for multiobjective problems (bi-objective in this work) are obtained by the $\varepsilon$-constrained approach, where only one of the objectives is treated as an objective function, while the other one is treated as an additional constraint.

\section{Results}

The MP-IDEA algorithm was applied to the sizing problem outlined in Section 3, using the multirotor model and battery discharge model described in Section 2 . The results for different values of $d_{T R G}$ are reported in Fig. 3. a for a payload weight $W_{p l}=0.5 \mathrm{~kg}$ in the $W_{p l} / W_{T O}$ vs $t_{H}$ plane. The front for $d_{T R G}=0$ corresponds to the simpler local survey VLOS scenario. No constraint on vehicle size is enforced, hence optimal configurations on the Pareto fronts are all planar ones. Constraints on total weight $W_{T O}$ and hovering time $t_{H}$ are not enforced at algorithm level, to increase its efficiency. Limits on $t_{H}$ and $W_{T O}$ are shown on the final plots, (red lines in Fig.3.a). The resulting region of feasible solutions is easily determined on the $W_{p l} / W_{T O}$ vs $t_{H}$ plane, where one can identify the best compromise between competing objectives. As an example, a limit of $10 \mathrm{~kg}$ on payload weight, corresponding to a value $W_{p l} / W_{T O}=0.05$, is represented by a horizontal red line. The bottom left corner of the feasible region thus identifies the vehicle configuration weighting less than $10 \mathrm{~kg}$, reaching the farthest distance (approximately $5 \mathrm{~km}$ ), with an almost zero hovering time at target. Increasing $t_{H}$ allows for a shorter distance to be flown. Maximum hovering time at $d_{T R G}=0$ for a $10 \mathrm{~kg}$ multirotor is in the order of 1 hour and 25 minutes.

It should be noted that values of $V_{1}$ and $V_{2}$ obtained on the Pareto fronts are very similar in all the considered test cases. This means that the nonlinearity induced by the battery discharge model has a limited impact on the best range cruise speed for the two cruise legs, to and from the target operational area. In order to reduce the number of design variables and obtain a numerically more efficient sizing tool, it will thus be possible to consider only one design variable for both cruise legs, namely cruise speed $V_{\text {cr. }}$.

A second parametric study was performed for a prescribed distance to target, $d_{T R G}=3 \mathrm{~km}$, and three different values of payload weight, namely $W_{p l}=0.1,0.5$, and $1.0 \mathrm{~kg}$. In this case the requirement for a positive hovering time on target is again represented by a vertical red line for $t_{H}=0$, in the $W_{p l} / W_{T O}$ vs $t_{H}$ plane, whereas the limit on maximum takeoff weight is now represented by the curve on the right of the plot. The region of feasible configurations lies in between. If on one side, the qualitative results appears as obvious, that is, a higher payload requires a heavier vehicle for reaching the same distance and/or flying the same time, 
the optimization tool provides a design that allows one to keep total weight down to a minimum and within prescribed limits. Also, the feasibility of a requirement is immediately evident, e.g. with the considered pieces of equipment it is not possible to fly more than approximately $5 \mathrm{~km}$, with vehicles weighting less than $10 \mathrm{~kg}$.
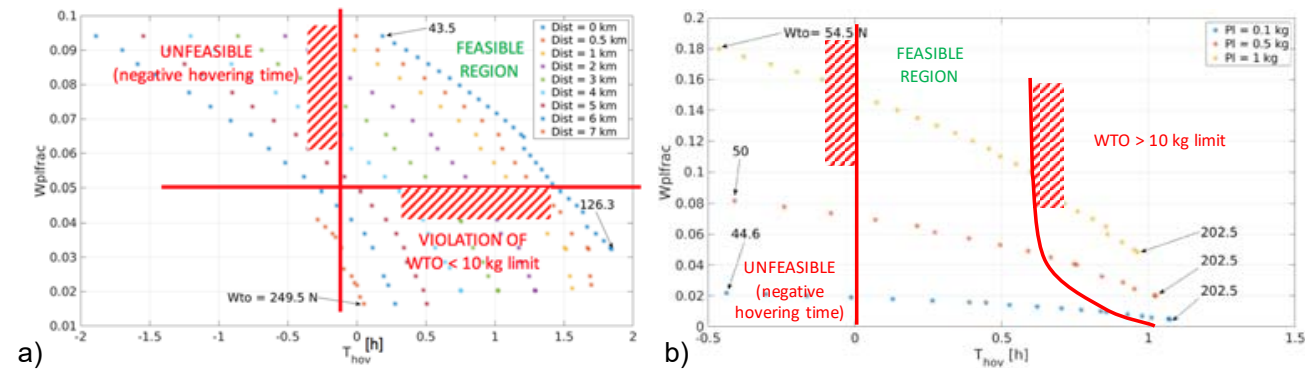

Fig. 3. Pareto fronts for (a) payload weight $W_{p l}=0.5 \mathrm{~kg}$ and different values of distance $d_{T R G}$ from target; (b); distance to target $d_{T R G}=3 \mathrm{~km}$ and different values of payload weight $W_{p l}$.

\section{Conclusions}

A memetic evolutionary algorithm MP-IDEA is applied to the problem of sizing electrically powered multirotor configurations. A standard multirotor vehicle model is coupled to a recent constant power discharge model, developed into a discharge model that accounts for step variations in power drawn from the batteries. The optimization tool proved to be efficient and robust for tackling the considered problem. Results at hovering are consistent with previous semi-analytical results for battery pack sizing. In the multi-objective design process, configuration tradeoffs are easily identified, together with relevant mission constraints and requirements, thus resulting in an overall mission feasibility analysis.

\section{References}

1. G. Avanzini, E.L. de Angelis, F. Giulietti, Aerosp. Sc. Tech. 59, 132-144 (2016)

2. E.L. de Angelis, G. Ferrarese, F. Giulietti, D. Modenini, P. Tortora, J. Guid, Cont. Dyn. 38, 9, 1827-1833 (2015)

3. E. L. de Angelis, G. Ferrarese, F. Giulietti, D. Modenini, P. Tortora, Aerosp. Sc. Tech. 55, 366-376 (2016)

4. L. Pollini, F. Giulietti, G. Avanzini, Proc. Inst. Mech. Eng., part G: J. Aerosp. Eng. 230, 174-179 (2016)

5. E.L. de Angelis, F. Giulietti, G. Rossetti, Aerosp. Sc. Tech. 77, 733-741 (2018)

6. E.L. de Angelis, Aerosp. Sc. Tech. 72, 248-255 (2018)

7. D. Raymer, Aircraft Design. A Conceptual Approach (5 Ed. AIAA, Reston, VA, 2012)

8. J. Roskam, Airplane Design Part I: Preliminary sizing of airplanes (DARcorporation, Lawrence, 1985)

9. E. Torenbeek, Synthesis of Subsonic Airplane Design (Delft University Press, Delft, 1976)

10. L. Traub, J. Aircraft 48, 2, 703-707 (2011)

11. G. Avanzini, F. Giulietti, J. Aircraft 50, 1, 304-307 (2013)

12. Avanzini, A. Carlà, T. Donateo, Proc. Inst. Mech. Eng., part G: J. Aerosp. Eng. 231, 2282-2294 (2017)

13. M. Gatti, F. Giulietti, M. Turci, Aerosp. Sc. Tech. 45, 174-179 (2015).

14. W. Peukert, Elektrotechnik 20, 20-21 (1897)

15. M. Di Carlo, M. Vasile, E. Minisci. Multi-Population Adaptive Inflationary Differential Evolution Algorithm with adaptive Local Restart. Proc CEC 2015. Sendai, Japan (2015) 